\title{
Model and Simulation of a Tunable Birefringent Fiber Using Capillaries Filled with Liquid Ethanol for Magnetic Quasiphase Matching In-Fiber Isolator
}

\author{
Clint Zeringue and Gerald T. Moore \\ Advanced Electric Laser Branch, Air Force Research Laboratory, Directed Energy Directorate, Kirtland Air Force Base, \\ NM 87117, USA \\ Correspondence should be addressed to Clint Zeringue, clint.zeringue@kirtland.af.mil
}

Received 30 November 2009; Revised 16 February 2010; Accepted 25 March 2010

Academic Editor: Iyad Dajani

Copyright ( $) 2010$ C. Zeringue and G. T. Moore. This is an open access article distributed under the Creative Commons Attribution License, which permits unrestricted use, distribution, and reproduction in any medium, provided the original work is properly cited.

A technique to tune a magnetic quasi-phase matching in-fiber isolator through the application of stress induced by two mutually orthogonal capillary tubes filled with liquid ethanol is investigated numerically. The results show that it is possible to "tune" the birefringence in these fibers over a limited range depending on the temperature at which the ethanol is loaded into the capillaries. Over this tuning range, the thermal sensitivity of the birefringence is an order-of-magnitude lower than conventional fibers, making this technique well suited for magnetic quasi-phase matching.

\section{Introduction}

Optical isolators play a critical role in the fabrication of highpower fiber amplifiers. Their purpose is to protect optical sources from light traveling in the backward direction which can cause damage to the optical source and instabilities in the output spectra. Optical isolators come in two flavors: polarization-dependent and polarization-independent. The polarization dependent isolator consists of an input polarizer, a Faraday rotator, and an output polarizer. Polarization independent isolators consist of an input birefringent wedge (with its ordinary polarization direction vertical and its extraordinary polarization direction horizontal), a Faraday rotator, and an output birefringent wedge oriented at $45^{\circ}$ to the first. Ideally the birefringent crystals and Faraday rotator should have low absorption coefficients at the wavelengths of interests, low nonlinear refractive indices, and high damage thresholds. Also, the Faraday rotator should have a high Verdet constant to achieve the highest degree of rotation with minimal length to prevent self-focusing inside the material and other thermal effects, as well as nonreciprocal nonlinear polarization coupling at high power.
The major sources of backward traveling light in highpower fiber lasers and amplifiers are reflections from the fiber output facet, spontaneous Raleigh scattering (SRS), stimulated Brillouin scattering (SBS), and amplified spontaneous emission (ASE). While certain techniques may be employed to minimize backward-traveling light, it is often a safe practice to incorporate isolators capable of suppressing backward power of at least $1-5 \%$ of the output power of the laser or amplifier. Current (free space) isolators can handle average powers up to the order of $100 \mathrm{~W}$ with limited beam distortions. For fiber-coupled devices, the power levels are currently limited to about $50 \mathrm{~W}$.

Recent demonstrations show continuous wave (CW) diffraction-limited power in fiber amplifiers scalable to the $\mathrm{kW}$ level [1]. These amplifiers are often seeded with powers approaching the current tolerances of fiber coupled isolators and thus are not suitable for high-power applications [2]. It is possible to use high-power free-space isolators to avoid these power limitations. However, these isolators tend to be very lossy, require precision alignment, and in these configurations the amplifier is not a monolithic system. Furthermore, fiber-coupled isolators are not truly all in-fiber isolators, 


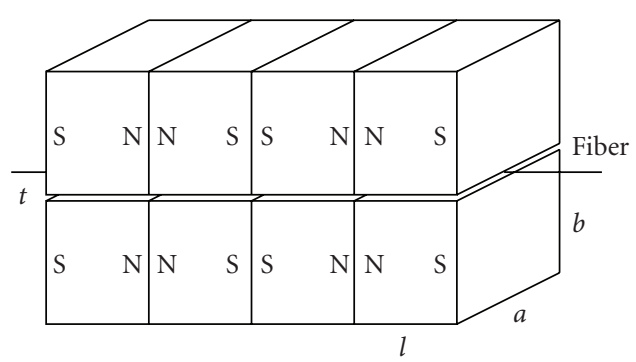

Figure 1: Example of quasi-phase matching technique. Two out of about 57 periods are shown.

since light leaves the fiber core to propagate through a thin Faraday rotator and small birefringent elements before coupling back into another fiber. As fiber amplifiers are scaled to the multikilowatt level, fiber isolators will be key components in the high-power system. Overcoming the power limitations associated with fiber-coupled isolators at the $100 \mathrm{~W}$ level is a tremendous challenge and creates a demand for novel concepts in optical isolation. One such concept uses a polarization-maintaining fiber with fairly low birefringence produced by solid stress rods and additional tunable birefringence produced by a secondary set of mutually orthogonal capillary tubes filled with liquid ethanol [2]. This fiber is used in conjunction with magnetic quasi-phase matching [3], where the polarization beat length at the wavelength of the fiber amplifier is matched to the period of an array of permanent magnets.

\section{Magnetic Quasi-Phase Matching}

2.1. Theoretical Concept. By matching the beat length of birefringent single-mode fiber to the period of a spatially alternating magnetic field within the fiber, $45^{\circ}$ Faraday rotation is achievable in undoped silica fiber in less than $1 \mathrm{~m}^{3}$. This configuration is shown in Figure 1. A fiber, or a set of similar parallel fibers for multiple isolators, is placed between two rows of magnets arranged with alternating polarities. In this configuration, the polarization beat length $L$ at the wavelength of light propagating in the fiber core is chosen to closely match the period $2 l$ of the magnetic array.

The local magnetic field at any point along the fiber is given by [3]:

$$
B_{N}(z)=\sum_{j=0}^{N-1}(-1)^{j+1} B_{1}(z-j l)
$$

where $B_{1}$ is the longitudinal field in the fiber produced by one magnet pair. Assuming ideal conditions for the polarizers, the transmissions of the quasi-phase matched isolator in the forward and reverse directions are [3]

$$
\begin{aligned}
& T_{\text {fwd }}=\frac{1}{2}\left(1+\sqrt{1-\Delta^{2}}\right), \\
& T_{\text {bwd }}=\frac{1}{2}\left(1-\sqrt{1-\Delta^{2}}\right),
\end{aligned}
$$

where,

$$
\Delta \equiv\left|E_{x}\right|^{2}-\left|E_{y}\right|^{2}=1-2\left(\frac{V \bar{B}}{\beta}\right)^{2} \sin ^{2} \beta z
$$

denotes the difference between polarization powers in the $x$ and $y$ directions.

Before entering the magnet array at $z=0$ the light passes through a polarizer (e.g., a $45^{\circ}$ tilted fiber Bragg grating). This polarizer is oriented to transmit the $x$ polarization and remove the $y$ polarization. At the entrance of the magnet array, the normalized field is $\left|E_{x}\right|=1$ and $\Delta=1$. The interplay of fiber birefringence and Faraday rotation under phase-matched conditions causes Faraday rotation to increase over the length of the array. The birefringence acts similarly as a succession of half-wave plates, producing reflections of the polarization about the fiber axes with the same periodicity as the magnetic field reversals. To make an isolator, one needs $\Delta=0$ at the end of the magnet array. There a second polarizer is affixed rotated by $45^{\circ}$ with respect to the fiber axes. By translating the fiber longitudinally within the magnet array, the polarization there is made linear and transmitted through the polarizer. However, backwardpropagating light with either polarization is blocked by one or the other of the two polarizers.

In the above equations, $V$ and $\bar{B}$ denote the Verdet constant of the fiber and the phase-matched Fourier component of the spatially oscillating magnetic field. $\beta$ is a function of the "detuning" parameter q:

$$
\begin{gathered}
\beta=\sqrt{q^{2}+(V \bar{B})^{2}}, \\
q=\pi\left(\frac{1}{2 l}-\frac{1}{L}\right) .
\end{gathered}
$$

The minimum magnetic field to achieve ideal isolation for a fiber length $l_{f}=N l$ is obtained by setting $\Delta=0, q=0$ and thus,

$$
\begin{gathered}
T_{\mathrm{fwd}}=1, \\
T_{\mathrm{bwd}}=0, \\
\bar{B}=\frac{\pi}{4 V l_{f}} .
\end{gathered}
$$

With the fields available from NdFeB permanent magnets, sufficient Faraday rotation at $1 \mu \mathrm{m}$ wavelength requires a magnet array about $1 \mathrm{~m}$ long. It would be practically impossible to achieve sufficient Faraday rotation in undoped fused silica using a single magnet.

The beat length $L$ is equal to the wavelength $\lambda$ divided by the fiber birefringence $B$. Phase matching occurs at only one wavelength. If the magnetic field exceeds the minimum given above, ideal isolation with $\Delta=0$ but $q \neq 0$ is possible at either of two wavelengths slightly above or below the phasematched wavelength. The wavelength range over which good isolation occurs scales inversely with the number of magnet periods. 


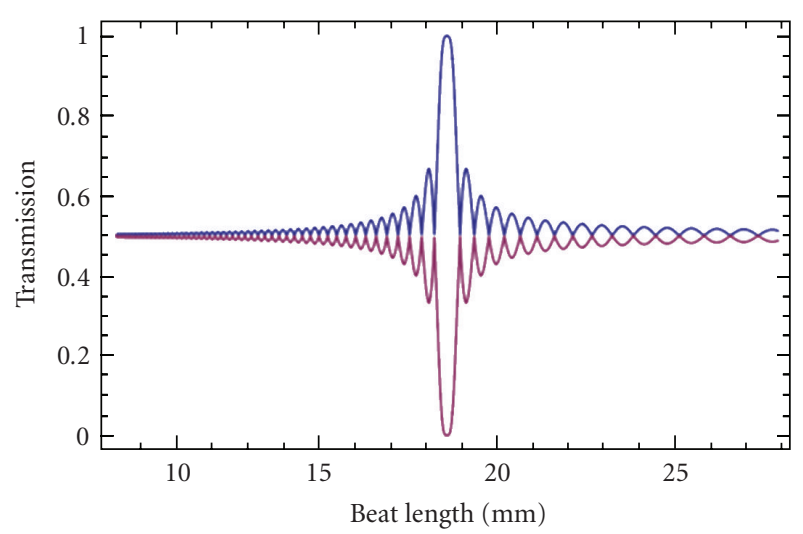

Figure 2: Backward (red) and Forward (blue) transmissions through in-fiber isolator using parameters of Table 1.

TABle 1: Practical parameters for Magnetic Quasi-Phase matching in a fiber.

\begin{tabular}{lc}
\hline$a$ & $24(\mathrm{~mm})$ \\
$l$ & $9.3(\mathrm{~mm})$ \\
$b$ & $12(\mathrm{~mm})$ \\
$t$ & $0.6(\mathrm{~mm})$ \\
$\bar{B}$ & $0.748 \mathrm{~T}$ \\
$V$ & 1.129 radians/Tm \\
$N$ & 100 \\
\hline
\end{tabular}

2.2. Considerations. In order for the all-fiber isolator to be effective in a practical system, care must be taken to match the beat length of the fiber to the period of the magnetic array in order to keep $q$ small. In order to characterize the effect on the in-fiber isolator when the beat length is not perfectly matched to the period of the magnetic array, it is useful to consider an example.

Using the parameters described in $[2,3]$ and also shown in Table 1, the backward and forward transmissions as a function of beat length $L$ through crossed polarizers were computed using (2).

As shown in Figure 2, a change of only $0.7 \mathrm{~mm}$ in the beat length reduces the transmission by $50 \%$ and thus precise control over the birefringence is a necessary requirement for a practical isolator. Conventional fibers are susceptible to temperature-induced changes in birefringence in the range $[4,5](1 / B)(\partial B / \partial T)=5-7 \times 10^{-4} \mathrm{~K}^{-1}$. For a wavelength of $1.064 \mu \mathrm{m}$ and assuming a modal birefringence of $1 \times$ $10^{-5}$ (typical in Low-Bi Fibers), fluctuations on the order of $10 \mathrm{C}$ would reduce the transmission through the crossed polarizers by $50 \%$. Thus, even in laboratory environments where the temperature in the room may fluctuate between $5-10^{\circ} \mathrm{C}$, reliable operation is unlikely.

Another consideration is isolation at multiple wavelengths. Traditional isolators have band passes on the order of $20 \mathrm{~nm}$. Changes in the isolation over the band pass are typically very small. However, using the magneticquasiphase matching technique, the situation is quite different. Again using a typical birefringence of $1 \times 10^{-5}$,



FIgURE 3: Example configuration of conventional fiber with a secondary set of mutually orthogonal capillary tubes filled with liquid ethanol.

there is approximately $2.4 \mathrm{~mm}$ difference between the beat lengths of $1064 \mathrm{~nm}$ and $1084 \mathrm{~nm}$; greater than the $0.7 \mathrm{~mm}$ limit mentioned previously. In order to overcome these impracticalities, one needs to be able to "tune" the birefringence to a specific wavelength and at the same time have a temperature-insensitive device. A solution to this problem was recently proposed [2] based on the inclusion of a secondary orthogonal set of liquid ethanol filled holes.

\section{Tunable Birefringence Using Pressurized Liquid Capillaries}

3.1. Overview. One possible technique to control the birefringence in a fiber is to insert a set of perpendicular liquid-filled holes mutually orthogonal to the traditional borosilicate stress rods in PANDA fiber as shown in Figure 3. The holes are filled with liquid ethanol because of its high thermal expansion coefficient and low freezing temperature.

In general, the birefringence in a fiber is given by

$$
B=C\left(\sigma_{x}-\sigma_{y}\right)
$$

where $C$ is the stress-optic coefficient and $\sigma_{x}$ and $\sigma_{y}$ denote the stress in the $x$ and $y$ directions respectively. The relationships between stress, strain, and thermal expansion are given by [6]:

$$
\begin{array}{ll}
\varepsilon_{x}=\frac{1}{E}\left[\sigma_{x}-v\left(\sigma_{y}+\sigma_{z}\right)\right]+\alpha \Delta T, & \gamma_{x y}=\frac{\partial u}{\partial y}+\frac{\partial v}{\partial x} \\
\varepsilon_{y}=\frac{1}{E}\left[\sigma_{y}-v\left(\sigma_{x}+\sigma_{z}\right)\right]+\alpha \Delta T, & \gamma_{x z}=\frac{\partial u}{\partial z}+\frac{\partial w}{\partial x} \\
\varepsilon_{z}=\frac{1}{E}\left[\sigma_{z}-v\left(\sigma_{x}+\sigma_{y}\right)\right]+\alpha \Delta T, & \gamma_{y z}=\frac{\partial v}{\partial z}+\frac{\partial w}{\partial y}
\end{array}
$$

where $\varepsilon_{x} \cdots \gamma_{y z}$ are called the components of strain. E, $\alpha$, and $\Delta T$ denote Young's Modulus, thermal expansion coefficient, and the change in temperature between the fiber draw temperature and operating temperature, respectively. 


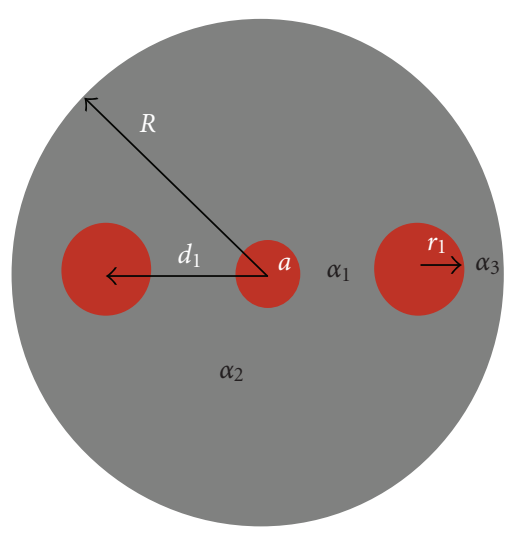

FIgURE 4: Example of standard (PANDA) fiber.

In general, the strain in each principal direction is related to the respective displacements $u, v$, and $w$ as

$$
\varepsilon_{x}=\frac{\partial u}{\partial x}, \quad \varepsilon_{y}=\frac{\partial v}{\partial y}, \quad \varepsilon_{z}=\frac{\partial w}{\partial z} .
$$

In standard (PANDA) fibers such as that shown in Figure 4 the magnitude of the birefringence is determined by several parameters. Referencing Figure $4, d_{1}$, and $r_{1}$ describe the distance between the stress applying parts and the size of the stress rods respectively. $a$ and $R$ denote the fiber core and fiber size. $\Delta T$ is the difference between the draw temperature of the fiber and the operating temperature. The thermal expansions coefficients of the core, cladding, and stress rods are $\alpha_{1}, \alpha_{2}$, and $\alpha_{3}$ respectively. Some other important parameters are Young's Modulus $(E)$, the stressoptic coefficient $(C)$, and Poisson's ratio $(v)$.

In the case of the plane-strain approximation, $\varepsilon_{z}=0$. The plane-strain approximation is appropriate for long fibers where the ends are rigid and frictionless. In this approximation, the birefringence may be obtained by analytic means. The total thermoelastic strain is related to the product $\alpha \Delta T$ across the fiber cross section by [7]:

$$
\begin{aligned}
& \frac{\partial e}{\partial x}+(1-2 v) \nabla^{2} u=2(1-v) \frac{\partial(\alpha \Delta T)}{\partial x} \\
& \frac{\partial e}{\partial y}+(1-2 v) \nabla^{2} v=2(1-v) \frac{\partial(\alpha \Delta T)}{\partial y} .
\end{aligned}
$$

Using the thermoelastic displacement potential,

$$
u=\frac{\partial \phi}{\partial x}, \quad v=\frac{\partial \phi}{\partial y} .
$$

The particular solution to (9) is obtained by Poisson's equation in each region:

$$
\begin{aligned}
& \nabla^{2} \varphi_{i}=\frac{1+v}{1-v}\left(\alpha_{i}-\alpha_{2}\right) \Delta T \quad \text { (inside the region), } \\
& \nabla^{2} \varphi_{i}=0 \quad \text { (outside the region) } \\
& \sum_{i} \varphi_{i}=\varphi .
\end{aligned}
$$

The complimentary solution is obtained by solving,

$$
\begin{aligned}
& \frac{\partial e}{\partial x}+(1-2 v) \nabla^{2} u=0 \\
& \frac{\partial e}{\partial y}+(1-2 v) \nabla^{2} v=0
\end{aligned}
$$

Applying continuity of the potential at the region boundaries and forcing $\sigma_{r}$ and $\sigma_{r \theta}$ to go to zero at $R$, the full analytic solution is obtained.

With the addition of the pressure introduced in the walls of the liquid filled stress rods, the boundary conditions across the interface between the capillary walls and the surrounding cladding are such that the pressure is continuous

$$
\sigma_{r=\text { hole boundaries }}=P(\rho, T),
$$

where $P(\rho, T)$ denotes the pressure on the walls as a function of density and temperature of the liquid ethanol. In this configuration the, symmetry of the problem is broken, and an analytic approach is unknown to these authors. For this reason, we approach this problem using the Finite Element Method (FEM), which is well suited for hyperbolic differential equations. Using the commercially available FEM package "Comsol", (7)-(9) were solved numerically in the plane-strain approximation on a triangular mesh with 13112 elements and 6651 mesh points with the additional boundary conditions given by (13).

The pressure on the boundary of the holes is determined using the empirical equation-of-state of liquid ethanol [810]:

$$
\begin{aligned}
& p-p_{o}=\sum_{i=1}^{3} \sum_{j=0}^{3} C_{i j}\left(\rho-\rho_{o}\right)^{i} T^{j} \\
& \rho=\rho_{o} /\left[1-\left(\sum_{i=1}^{3} A_{i} T^{i}\right) \ln \left(1+p \sum_{j=1}^{3} B_{j} T^{j}\right)\right], \\
& \rho_{o}=806.594-0.84589 T_{\text {load }} \\
& \quad-1.361 * 10^{-4} T_{\text {load }^{2}}-5.557 * 10^{-6} T_{\mathrm{load}^{3}}{ }^{3}
\end{aligned}
$$

where $p, p_{o}, \rho, \rho_{o}$, and $T, T_{\text {load }}$ denote pressure, reference pressure, density, reference density, operating temperature, and loading temperature, respectively.

3.2. Methodology. In general, the average birefringence of the core as well as the sensitivity of the birefringence to temperature is dependent on the several factors mentioned in the preceding section. Referencing Figure 5 in the fiber with the secondary set of liquid rods, two additional factors are introduced: $d_{2}$, the distance from the center of the fiber to the liquid rod center, and the loading temperature of the liquid ethanol.

In an effort to limit the parameter space, we fix the core and cladding diameter to $25 \mu \mathrm{m}$ and $200 \mu \mathrm{m}$, respectively. Furthermore, we restrict the loading temperature of the liquid ethanol to a span of $-80^{\circ} \mathrm{C}$ to $40^{\circ} \mathrm{C}$ and assume a draw temperature of $1073^{\circ} \mathrm{C}$. We then generated a large 


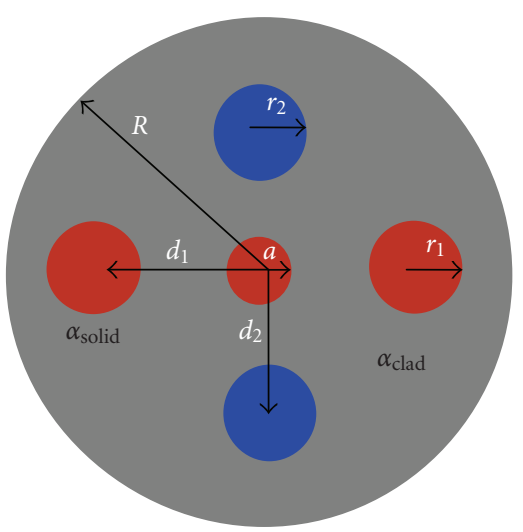

$\mathrm{C}_{2} \mathrm{H}_{5} \mathrm{OH}$
$\mathrm{B}_{2} \mathrm{O}_{3}+\mathrm{SiO}_{2}$
$\mathrm{SiO}_{2}$

Figure 5: Conventional fiber with a secondary set of mutually orthogonal capillary tubes filled with liquid ethanol.

Table 2: Practical parameters for Magnetic Quasi-Phase matching in a fiber.

\begin{tabular}{|c|c|c|}
\hline Name & Expression & Description \\
\hline$R$ & $0.0001(\mathrm{~m})$ & Fiber Radius \\
\hline$T_{\text {melt }}$ & $1346.15(\mathrm{~K})$ & Fiber Melting Temperature \\
\hline E & $73 \mathrm{e} 9(\mathrm{~Pa})$ & Young's Modulus of Fiber \\
\hline$\alpha_{\text {solid }}$ & $2.28 \mathrm{e}-006(1 / \mathrm{K})$ & $\begin{array}{l}\text { Thermal Expansion Coefficient of solid } \\
\text { rods }\end{array}$ \\
\hline$\alpha_{\text {clad }}$ & $5 e-007(1 / K)$ & $\begin{array}{l}\text { Thermal Expansion Coefficient of } \\
\text { Cladding }\end{array}$ \\
\hline$v$ & $0.17(1)$ & Poison's Ratio of Fiber \\
\hline$a$ & $1.25 \mathrm{e}-005(\mathrm{~m})$ & Core Radius \\
\hline C & $3.22 \mathrm{e}-012(1 / \mathrm{Pa})$ & Stress-Optic Coefficient at $1064 \mathrm{~nm}$ \\
\hline
\end{tabular}

sample space for the parameters $r_{1}, r_{2}, d_{1}, d_{2}$ and $T_{\text {load. }}$. For each set, the average birefringence and sensitivity of the birefringence with respect to temperature were computed over the fiber core. The objective was to find parameters such that $(1 / \mathrm{B})(\partial \mathrm{B} / \partial \mathrm{T})$ was as small as possible (insensitive to temperature effects over the loading temperature range) while at the same time offering a large tune-ability in the beat length with respect to the loading temperature of the ethanol $\partial \mathrm{Beat} / \partial \mathrm{T}_{\text {load }}$. A further restriction was to limit the beat length at $\lambda=1.064 \mu \mathrm{m}$ to approximately $1.7 \mathrm{~cm}$ near room temperature. The relevant parameters and constants used in the following simulations are given in Table 2.

3.3. Results and Discussion. As described in the proceeding sections the parameters $r_{1}, r_{2}, d_{1}, d_{2}$ and $T_{\text {loadwere inves- }}$ tigated. The approach was to minimize the sensitivity in the birefringence with respect to operating temperatures near room temperature while maximizing the sensitivity in the beat length with respect to loading temperature.

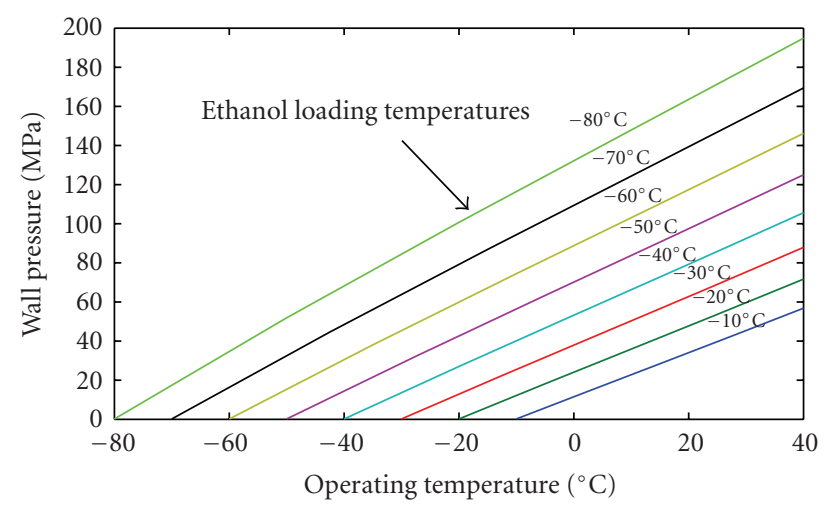

Figure 6: Wall Pressure versus operating temperature of capillary tubes filled with liquid ethanol.

TAble 3: Practical parameters for Magnetic Quasi-Phase matching in a fiber.

\begin{tabular}{lcc}
\hline Name & Expression & Description \\
\hline$R$ & $0.0001(\mathrm{~m})$ & Fiber Radius \\
$d_{1}$ & $3.46 \mathrm{e}-005(\mathrm{~m})$ & Center-to-center distance of solid rods \\
$d_{2}$ & $4.78 \mathrm{e}-005(\mathrm{~m})$ & Center-to-center distance of solid rods \\
$r_{1}$ & $8.2 \mathrm{e}-006(\mathrm{~m})$ & Solid rod radius \\
$r_{2}$ & $2.9 \mathrm{e}-006(\mathrm{~m})$ & Liquid rod radius \\
$a$ & $1.25 \mathrm{e}-005(\mathrm{~m})$ & Core Radius \\
\hline
\end{tabular}

The "tune ability" of the birefringence is dictated by the pressure exerted by the liquid ethanol on the walls of the capillary tubes. In this model, the liquid-filled capillaries are assumed to be loaded at atmospheric pressure and a loading temperature spanning from $-80^{\circ} \mathrm{C}$ to $40^{\circ} \mathrm{C}$. The capillaries are assumed to be sealed after loading such that the density remains constant and is given by (15). A plot of the pressure on the boundary of the liquid-filled holes versus operating temperature is shown in Figure 6. The radial pressures considered here span a range of 0-200 MPa and are well within the Bulk Modulus of fused silica $~ 30 \mathrm{GPa}$.

Near room temperature, the wall pressure can be "tuned" from approximately 30-160 $\mathrm{MPa}$, resulting in a range of stresses applied in the orthogonal directions relative to the standard borosilicate rods. Figure 7 shows a plot of the birefringence resulting from stress contributions from both the solid and liquid filled rods with the parameters shown in Table 3. Notice the strong contribution of stress from the solid rods relative to the stress induced from the pressure on the capillary walls. In this way, the liquid filled ethanol provides fine tuning, allowing precise tunability based on the loading temperature of the ethanol.

Another important feature of Figure 7 is that the birefringence is uniform over the central core region, an important design consideration for a birefringent fiber.

After considering a significant portion of the parameter space, the fiber described by the parameters in Table 3 contained the largest potential for tunability, while at 


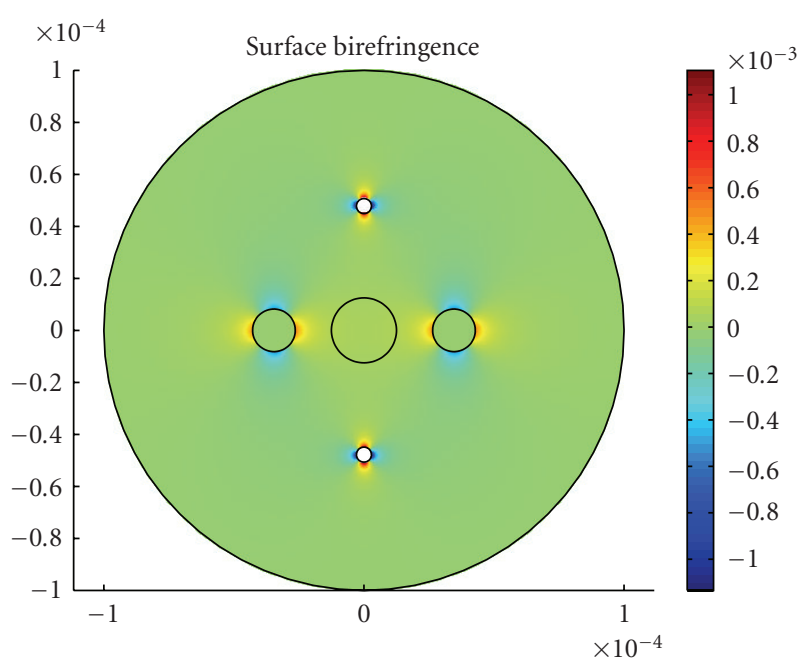

Figure 7: Example of birefringence of a fiber with both liquid and solid stress rods. The liquid capillary tubes and solid stress rods are shown aligned with the vertical and horizontal axes respectively.

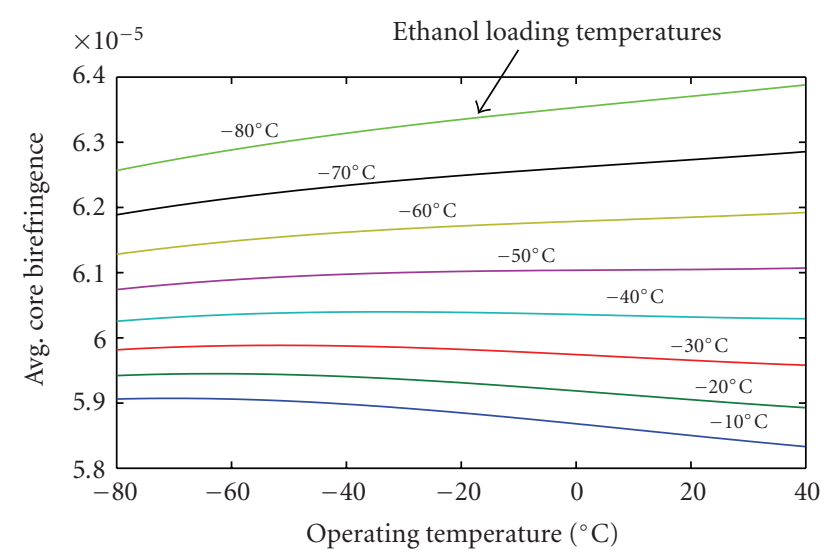

Figure 8: Average Core Birefringence versus Operating Temperatures.

the same time maintaining temperature insensitivity near room temperature. Figures 8 and 9 show plots of the average core birefringence and the sensitivity of the birefringence with respect to different operating temperatures.

Notice that in the normal operating regime $\left(20-40^{\circ} \mathrm{C}\right)$ the susceptibility of temperature-induced changes in birefringence is minimal $(1 / B)(\partial B / \partial T) \sim 10^{-5} \mathrm{~K}^{-1}$ for loading temperatures spanning $(-65 \cdots-35)^{\circ} \mathrm{C}$. This is nearly an order of magnitude smaller than that of the quoted number for standard fibers mentioned previously: $1 / \mathrm{B} \partial \mathrm{B} / \partial \mathrm{T}=5-7$ $\times 10^{-4} \mathrm{~K}^{-1}$

Figure 10 shows the corresponding beat length for this fiber using a wavelength of $\lambda=1.064 \mu \mathrm{m}$. Notice that over the same operating regime $\left(20-40^{\circ} \mathrm{C}\right)$, the beat length may be "tuned" from $(1.68-1.82) \mathrm{cm}$; or approximately $2 \mathrm{~mm}$, close to the $2 \mathrm{~mm}$ required and mentioned previously to have an isolator tunable over $20 \mathrm{~nm}$.

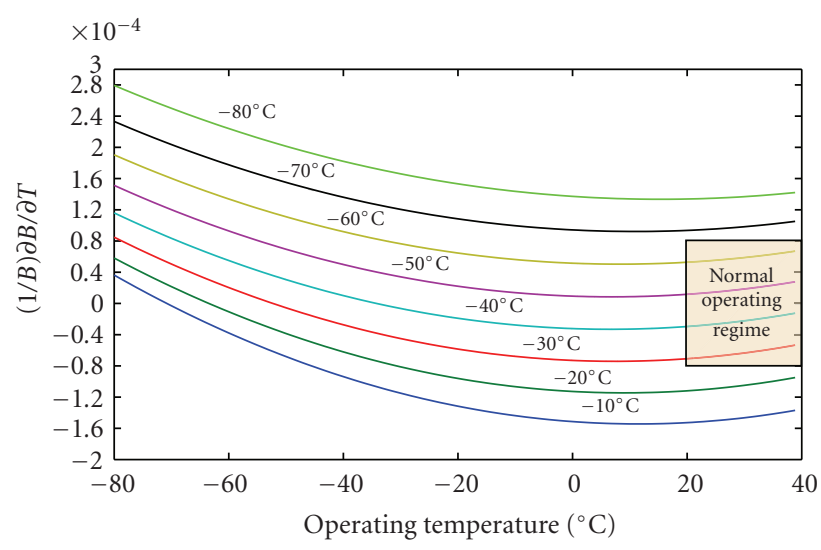

FIGURE 9: Normalized change in birefringence with respect to temperature versus operating temperature.

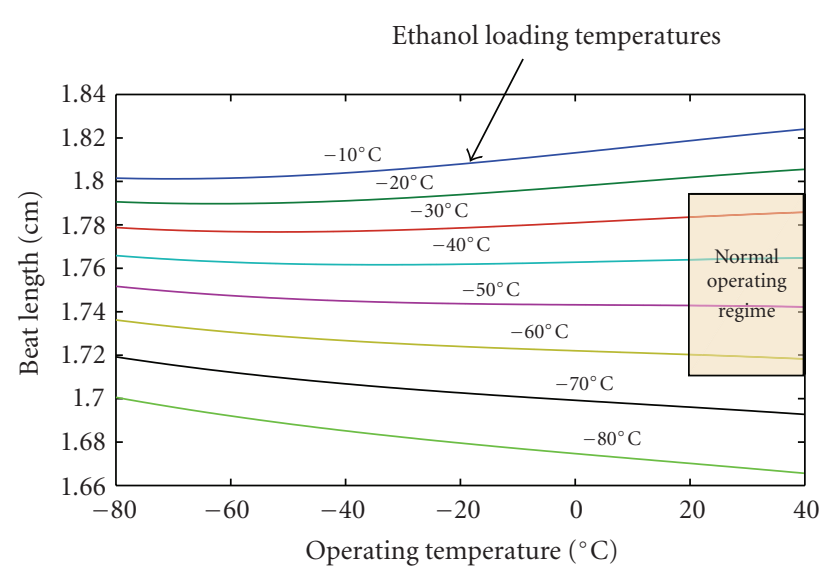

FIGURE 10: Beat length versus operating temperature for various ethanol loading temperatures.

\section{Conclusion}

A tunable, temperature insensitive, birefringent fiber was theoretically designed by investigating the use of a secondary set of mutually orthogonal liquid-filled capillaries in conjunction with traditional borosilicate stress rods. Although this paper is concerned with using this technique to construct a fiber with a nominal beat length of $1.75 \mathrm{~cm}$, a similar analysis could be done aiming at designs spanning a range of beat lengths. This paper has demonstrated that, by using a secondary set of capillaries filled with liquid ethanol, two of the practical limitations facing an all-fiber isolator using magnetic quasi-phase matching can be overcome: tunability is enabled to a selected wavelength within a range of $\sim 20 \mathrm{~nm}$ and temperature-insensitive birefringence is obtainable.

\section{Acknowledgments}

The authors would like to thank Iyad Dajani for useful discussions. This research was supported in part by the HighEnergy Laser Joint Technology Office. 


\section{References}

[1] Y. Jeong, J. K. Sahu, D. N. Payne, and J. Nilsson, "Ytterbiumdoped large-core fiber laser with $1.36 \mathrm{~kW}$ continuous-wave output power," Optics Express, vol. 12, no. 25, pp. 6088-6092, 2004.

[2] G. T. Moore, "Optical fiber with tunable birefringence using pressurized liquid capillaries," US Patent 7489847, February 2009.

[3] G. T. Moore and C A. Lu, in Proceedings of the Solid-State and Diode Laser Technology Review, Albuquerque, Mex, USA, June 2008.

[4] A. Michie, J. Canning, K. Lyytikäinen, M. Åslund, and J. Digweed, "Temperature independent highly birefringent photonic crystal fibre," Optics Express, vol. 12, no. 21, pp. 5160-5165, 2004.

[5] Z. B. Ren, P. Robert, and P.-A. Paratte, "Temperature dependence of bend- and twist-induced birefringence in a lowbirefringence fiber," Optics Letters, vol. 13, pp. 62-64, 1988.

[6] S. P. Timoshenkgo and J. N. Goodier, Theory of Elasticity, McGraw-Hill, New York, NY, USA, 1970.

[7] P. L. Chu and R. A. Sammut, "Analytical method for calculation of stresses and material birefringence in polarizationmaintaining optical fiber," Journal of Lightwave Technology, vol. 2, no. 5, pp. 650-662, 1984.

[8] T. F. Sun, J. A. Schouten, and S. N. Biswas, "Determination of the thermodynamic properties of liquid ethanol from 193 to $263 \mathrm{~K}$ and up to $280 \mathrm{MPa}$ from speed-of-sound measurements," International Journal of Thermophysics, vol. 12, no. 2, pp. 381-395, 1991.

[9] H. E. Dillon and S. G. Penoncello, "A fundamental equation for the calculation of the thermodynamic properties of ethanol," in Proceedings of the 15th Symposium on Thermophysical Properties, Boulder, Colo, USA, June 2003.

[10] S. A. Klein, "Engineering Equation Solver," Computer Software, F-Chart Software, Version 7.693, 2006. 

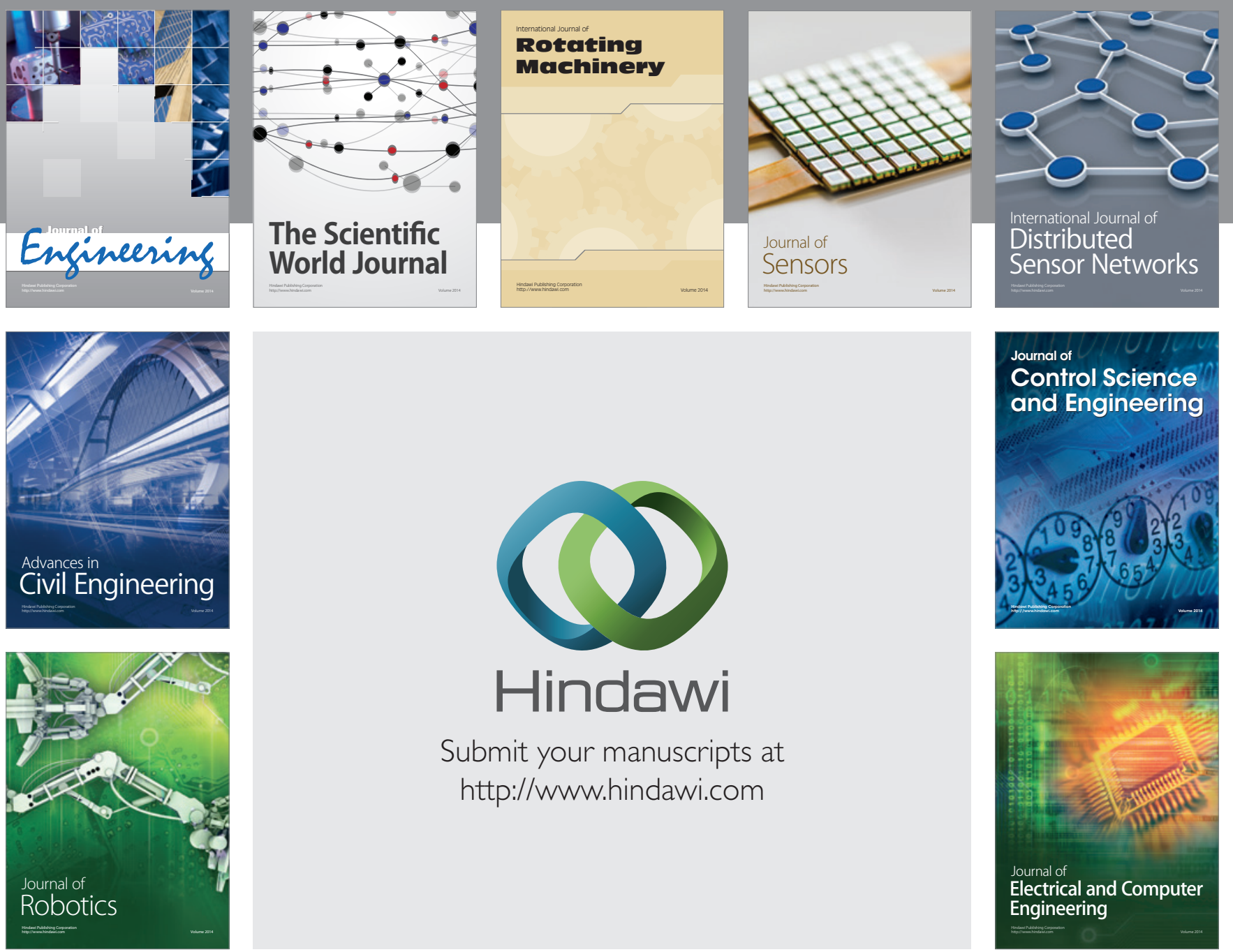

Submit your manuscripts at

http://www.hindawi.com
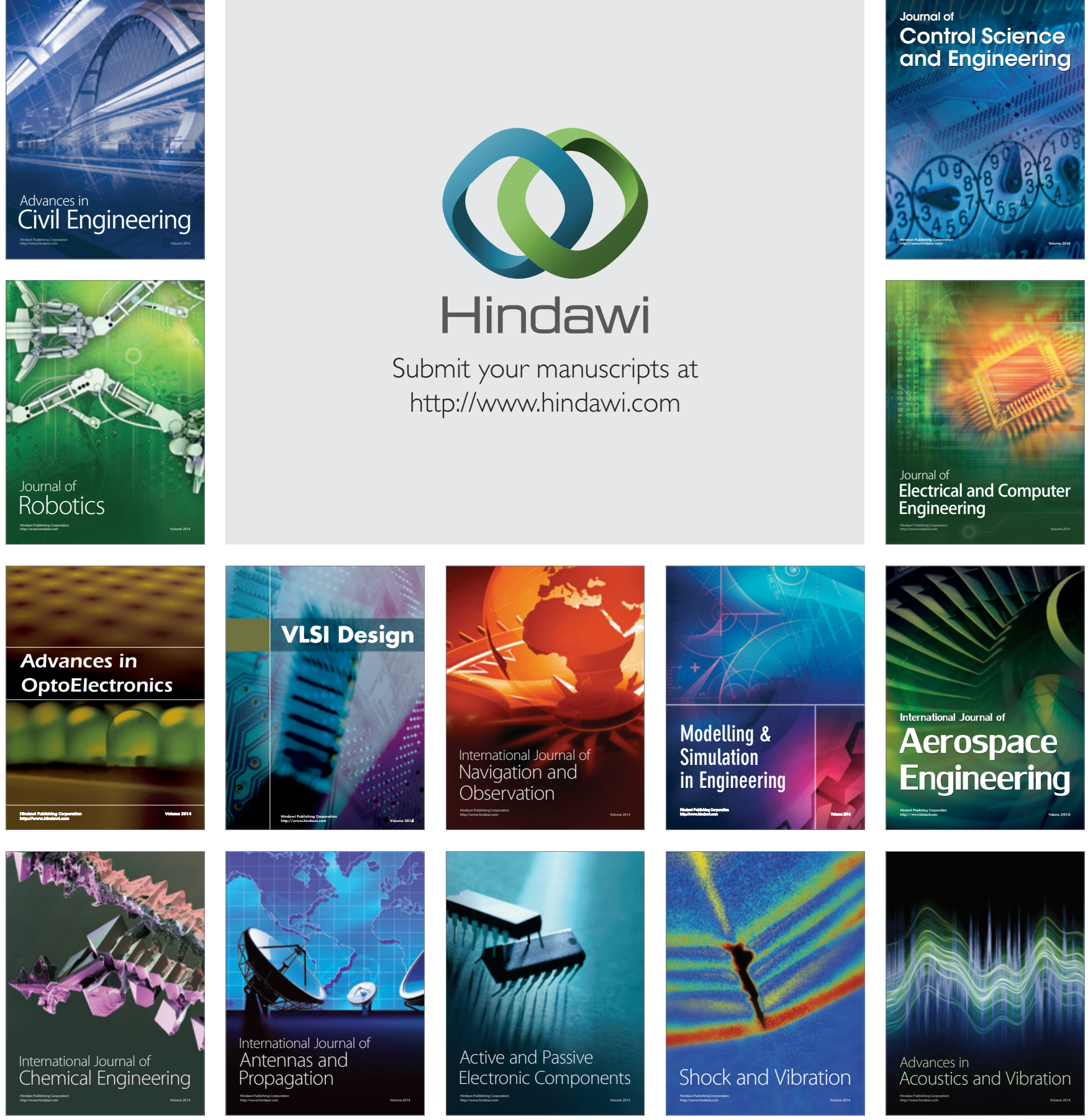